\title{
Some approximation results on Otto Szậsz type positive linear operators
}

\author{
Dr. R .P. Pathak and Shiv Kumar Sahoo \\ Department of Mathematics \\ National Institute of Technology, Raipur \\ G.E. Road, Raipur-492010 (C.G.) India.
}

ABSTRACT : Recently Deo N.et.al. (Appl. Maths. Compt., 201(2008), 604-612.) introduced a new Bernstein type special operators. Motivated by Deo N.et.al., in this paper we introduce generalization of positive linear operators (1.5) and (1.6) which is the particular case of positive linear operators (1.7) and (1.8). We shall study some approximation results on it.

\section{INTRODUCTION}

Recently Deo N.et.al. [1] introduced a new Bernstein type special operators $\left\{\boldsymbol{V}_{n} f\right\}$ defined as,

where

$$
\begin{gathered}
\left(V_{n} f\right)(x)=\sum_{k=0}^{n} p_{n, k}(x) f\left(\frac{k}{n}\right) \\
p_{n, k}(x)=\left(1+\frac{1}{n}\right)^{n}\left(\begin{array}{l}
n \\
k
\end{array}\right) x^{k}\left(\frac{n}{n+1}-x\right)^{n-k} ; \quad \text { for } 0 \leq x \leq \frac{n}{n+1}
\end{gathered}
$$

Again Deo N.et.al. [1] gave the integral modification of the operators (1.1) which are defined as ,

$$
\left(L_{n} f\right)(x)=n\left(1+\frac{1}{n}\right)^{2} \sum_{k=0}^{n} p_{n, k}(x) \int_{0}^{\frac{n}{n+1}} p_{n . k}(t) f(t) d t
$$

and prove some approximation results on the operators (1.2).

Singh S.P. [4] studied some approximation results on a sequence of Szậsz type operators defined as,

$$
\begin{aligned}
\left(S_{n, x} f\right)(t) & =\sum_{k=0}^{\infty} b_{n, k}(t) f\left(x+\frac{k}{n}\right) ; \\
\text { where } \quad b_{n, k}(t) & =e^{-n t} \frac{(n t)^{k}}{k !} ; \quad x \in[0, \infty) \text { is fixed. }
\end{aligned}
$$

which map the space of bounded continuous funtions $C_{B}[0, \infty)$ into itself following [3].

Kasana H.S. et. el. [2] obtained a sequence of modified Szậsz operators for integrable function on $[0, \infty)$ defined as,

$$
\begin{aligned}
\left(M_{n, x} f\right)(t) & \equiv M_{n, x}(f(y) ; t) \\
& =n \sum_{k=0}^{\infty} b_{n, k}(t) \int_{0}^{\infty} b_{n . k}(y) f(x+y) d y
\end{aligned}
$$

where $t, x \in[0, \infty)$ and $x$ is fixed.

Motivated by Deo N.et.al.[1] we studied a sequence of positive linear operators $\left\{\boldsymbol{B}_{n} f\right\}$ which are defined as,

where

$$
\left(B_{n} f\right)(x)=n\left(1+\frac{1}{n}\right)^{2} e^{-(n+p) x} \sum_{k=0}^{\infty} \frac{(n+p)^{k} x^{k}}{k !} \int_{0}^{\frac{n}{n+1}} p_{n . k}(t) f(t) d t
$$

we studied some approximation results on the operators (1.5).

Again following Kasana H.S. et. el. [2] we introduce a sequence of positive linear operators $\left\{\boldsymbol{B}_{n, x} f\right\}$ which are defined as,

$$
\left(B_{n, x} f\right)(t)=n\left(1+\frac{1}{n}\right)^{2} e^{-(n+p) t} \sum_{k=0}^{\infty} \frac{(n+p)^{k} t^{k}}{k !} \int_{0}^{\frac{n}{n+1}} p_{n . k}(y) f(x+y) d y
$$


where $t, x \in\left[0, \frac{n}{n+1}\right]$ and $x$ is fixed.

and studied some approximation results on the operators (1.6).

If we put $p=0$ in the operators (1.5) and (1.6), it gives the new modified operators $\left\{\boldsymbol{B}_{n}^{*} f\right\}$ and $\left\{\boldsymbol{B}_{n, x}^{*} f\right\}$ as:

$$
\left(B_{n}^{*} f\right)(x)=n\left(1+\frac{1}{n}\right)^{2} e^{-n x} \sum_{k=0}^{\infty} \frac{(n x)^{k}}{k !} \int_{0}^{\frac{n}{n+1}} p_{n . k}(t) f(t) d t
$$

and

$$
\begin{gathered}
\left(B_{n, x}^{*} f\right)(t)=n\left(1+\frac{1}{n}\right)^{2} e^{-n t} \sum_{k=0}^{\infty} \frac{(n t)^{k}}{k !} \int_{0}^{\frac{n}{n+1}} p_{n . k}(y) f(x+y) d y \\
\text { where } t, x \in\left[0, \frac{n}{n+1}\right] \text { and } x \text { is fixed. }
\end{gathered}
$$

We shall study some approximation results on the operators (1.7) and (1.8).

\section{BASIC RESULTS-I}

In order to prove our main result, the following basic results are needed.

$$
\begin{array}{lll}
\text { 1. } e^{-n x} \sum_{k=0}^{\infty} \frac{n^{k} x^{k}}{k !} k & =n x \\
\text { 2. } e^{-n x} \sum_{k=0}^{\infty} \frac{n^{k} x^{k}}{k !} k^{2} & =n^{2} x^{2}+n x \\
\text { 3. } e^{-n x} \sum_{k=0}^{\infty} \frac{n^{k} x^{k}}{k !} k^{3} & =n^{3} x^{3}+3 n^{2} x^{2}+n x \\
\text { 4. } e^{-n x} \sum_{k=0}^{\infty} \frac{n^{k} x^{k}}{k !} k^{4} & =n^{4} x^{4}+6 n^{3} x^{3}+7 n^{2} x^{2}+n x
\end{array}
$$

We know that

\section{PROOF OF BASIC RESULTS-I}

$$
e^{n x}=\sum_{k=0}^{\infty} \frac{(n x)^{k}}{k !}
$$

Differentiating with respect to $x$, we get

Multiplying $x$ both sides, we get

$$
n e^{n x}=\sum_{k=0}^{\infty} \frac{n^{k} k x^{k-1}}{k !}
$$

$$
\begin{gathered}
n x e^{n x}=\sum_{k=0}^{\infty} \frac{n^{k} x^{k}}{k !} k \\
n x=e^{-n x} \sum_{k=0}^{\infty} \frac{n^{k} x^{k}}{k !} k
\end{gathered}
$$

This completes the proof of (2.1).

Again differentiating (2.6) with respect to $x$, we get

$$
n^{2} x e^{n x}+n e^{n x}=\sum_{k=0}^{\infty} \frac{n^{k} k x^{k-1}}{k !} k
$$

Multiplying $x$ both sides, we get

This completes the proof of (2.2).

$$
\begin{aligned}
& n^{2} x^{2} e^{n x}+n x e^{n x}=\sum_{k=0}^{\infty} \frac{n^{k} x^{k}}{k !} k^{2} \\
& {\left[n^{2} x^{2}+n x\right] e^{n x}=\sum_{k=0}^{\infty} \frac{n^{k} x^{k}}{k !} k^{2} } \\
& {\left[n^{2} x^{2}+n x\right]=e^{-n x} \sum_{k=0}^{\infty} \frac{n^{k} x^{k}}{k !} k^{2} }
\end{aligned}
$$

In the same way after differentiations and calculations, we get required result s (2.3) and (2.4).

1. $\left(B_{n}^{*} 1\right)(x)=1$

\section{BASIC RESULTS-II}

www.iosrjournals.org 
2. $\left(B_{n}^{*} t\right)(x) \rightarrow x \quad$ as $n \rightarrow \infty$.

3. $\left(B_{n}^{*} t^{2}\right)(x) \rightarrow x^{2}$ as $n \rightarrow \infty$.

4. $\quad\left(B_{n}^{*} t^{3}\right)(x)=\frac{n^{3}\left[n^{3} x^{3}+9 n^{2} x^{2}+18 n x+6\right]}{(n+1)^{3}(n+2) \quad(n+3) \quad(n+4)}$

5. $\left(B_{n}^{*} t^{4}\right)(x)=\frac{n^{4}\left[n^{4} x^{4}+16 n^{3} x^{3}+72 n^{2} x^{2}+96 n x+24\right]}{(n+1)^{4} \quad(n+2) \quad(n+3) \quad(n+4) \quad(n+5)}$

6. $\left(B_{n}^{*}(t-x)\right)(x)=\frac{n[1-3 x]-2 x}{(n+1)(n+2)}$

7. $\left(B_{n}^{*}(t-x)^{2}\right)(x)=\frac{n^{3}\left(2 x-x^{2}\right)+n^{2}\left[11 x^{2}-8 x+2\right]+n\left[17 x^{2}-6 x\right]+6 x^{2}}{(n+1)^{2}(n+2)(n+3)}$

8. $\left(B_{n}^{*}(t-x)^{3}\right)(x)=o\left(\frac{1}{n}\right)$.

9. $\left(B_{n}^{*}(t-x)^{4}\right)(x)=o\left(\frac{1}{n^{2}}\right)$

Proof of Basic Results-II.

By putting $f(t)=1$ in equation (1.7), we get

$$
\begin{aligned}
\left(B_{n}^{*} 1\right)(x) & =n\left(1+\frac{1}{n}\right)^{2} e^{-n x} \sum_{k=0}^{\infty} \frac{n^{k} x^{k}}{k !} \int_{0}^{\frac{n}{n+1}}\left(\frac{n+1}{n}\right)^{n}\left(\begin{array}{l}
n \\
k
\end{array}\right) t^{k}\left(\frac{n}{n+1}-t\right)^{n-k} 1 d t \\
= & n\left(1+\frac{1}{n}\right)^{2} e^{-n x} \sum_{k=0}^{\infty} \frac{n^{k} x^{k}}{k !} \frac{1}{n}\left(\frac{n}{n+1}\right)^{2} \\
= & e^{-n x} \sum_{k=0}^{\infty} \frac{n^{k} x^{k}}{k !}=1 .
\end{aligned}
$$

This completes the proof of (2.8).

By putting $f(t)=t$ in equation (1.7), we get

$\left(B_{n}^{*} t\right)(x) \rightarrow x \quad$ as $\quad n \rightarrow \infty$.

$$
\begin{aligned}
\left(B_{n}^{*} t\right)(x) & =n\left(1+\frac{1}{n}\right)^{2} e^{-n x} \sum_{k=0}^{\infty} \frac{n^{k} x^{k}}{k !} \int_{0}^{\frac{n}{n+1}}\left(\frac{n+1}{n}\right)^{n}\left(\begin{array}{l}
n \\
k
\end{array}\right) t^{k}\left(\frac{n}{n+1}-t\right)^{n-k} t d t \\
& =n\left(1+\frac{1}{n}\right)^{2} e^{-n x} \sum_{k=0}^{\infty} \frac{n^{k} x^{k}}{k !} \frac{(k+1)}{n(n+2)}\left(\frac{n}{n+1}\right)^{3} \\
& =\frac{n}{(n+1)(n+2)}\left\{e^{-n x} \sum_{k=0}^{\infty} \frac{n^{k} x^{k}}{k !} k+e^{-n x} \sum_{k=0}^{\infty} \frac{n^{k} x^{k}}{k !} 1\right\} \\
& =\frac{n}{(n+1)(n+2)}[n x+1] \\
= & \frac{n^{2} x+n}{(n+1)(n+2)}
\end{aligned}
$$

This completes the proof of (2.9).

By putting $f(t)=t^{2}$ in equation (1.7), we get

$$
\begin{aligned}
& \left(B_{n} t^{2}\right)(x)=n\left(1+\frac{1}{n}\right)^{2} e^{-n x} \sum_{k=0}^{\infty} \frac{n^{k} x^{k}}{k !} \int_{0}^{\frac{n}{n+1}}\left(\frac{n+1}{n}\right)^{n}\left(\begin{array}{l}
n \\
k
\end{array}\right) t^{k}\left(\frac{n}{n+1}-t\right)^{n-k} t^{2} d t \\
& =n\left(1+\frac{1}{n}\right)^{2} e^{-n x} \sum_{k=0}^{\infty} \frac{n^{k} x^{k}}{k !} \frac{(k+1)(k+2)}{n(n+2)(n+3)}\left(\frac{n}{n+1}\right)^{4} \\
& =\frac{n^{2}}{(n+1)^{2}(n+2)(n+3)}\left\{e^{-n x} \sum_{k=0}^{\infty} \frac{n^{k} x^{k}}{k !} k^{2}+e^{-n x} \sum_{k=0}^{\infty} \frac{n^{k} x^{k}}{k !} 3 k+e^{-n x} \sum_{k=0}^{\infty} \frac{n^{k} x^{k}}{k !} 2\right\} \\
& =\frac{n^{2}\left[n^{2} x^{2}+4 n x+2\right]}{(n+1)^{2}(n+2)(n+3)}
\end{aligned}
$$

$\left(B_{n}^{*} t^{2}\right)(x) \rightarrow x^{2}$ as $n \rightarrow \infty$.

This completes the proof of (2.10). 
In the same way by taking $f(t)=t^{3} \& f(t)=t^{4}$ respectively in (1.7) and after little calculations we get required results $(2.11)$ to $(2.16)$.

This completes the proof.

\section{MAIN RESULTS}

In this section we shall give our main results

LEMMA: Let $f \in \mathcal{C}\left[0, \frac{n}{n+1}\right]$ then the sequence of positive linear operator defined by $\left\{B_{n}^{*} f\right\}$ is converges uniformly to $f$ as $n \rightarrow \infty$.

Proof : Since from basic results (2.8), (2.9) and (2.10), we get

Then using Korokin theorem we can conclude that

$$
\begin{aligned}
\left(B_{n}^{*} 1\right)(x) \stackrel{\text { uniformly }}{\longrightarrow} 1 \text { as } & n \rightarrow \infty \\
\left(B_{n}^{*} t\right)(x) \stackrel{\text { uniformly }}{\longrightarrow} x \text { as } & n \rightarrow \infty \\
\left(B_{n}^{*} t^{2}\right)(x) \stackrel{\text { uniformly }}{\longrightarrow} x^{2} \text { as } & n \rightarrow \infty
\end{aligned}
$$

This completes the proof.

$$
\left(B_{n}^{*} f\right)(x) \stackrel{\text { uniformly }}{\longrightarrow} f \text { as } \quad n \rightarrow \infty .
$$

Theorem : Let $f$ be the integrable and bounded in the interval $\left[0, \frac{n}{n+1}\right]$ and let if $f$, 'exists at a point $x$ in $\left[0, \frac{n}{n+1}\right]$, then one gets that

where $\left\{B_{n}^{*} f\right\}$ are defined in (1.7).

$$
\lim _{n \rightarrow \infty} n\left[\left(B_{n}^{*} f\right)(x)-f(x)\right]=(1-3 x) f^{\prime}(x)+\frac{x(2-x)}{2} f^{\prime \prime}(x) .
$$

Proof: Since $f$ ', exists at a point $x$ in $\left[0, \frac{n}{n+1}\right]$, then by using Taylor's expansion, we write

where $\lambda(t-x) \rightarrow 0$ as $t \rightarrow x$.

$$
f(t)=f(x)+(t-x) f^{\prime}(x)+\frac{(t-x)^{2}}{2} f^{\prime \prime}(x)+(t-x)^{2} \lambda(t-x)
$$

Now for each $\varepsilon>0$, there corresponds $\delta>0$ such that

$$
|\lambda(t-x)| \leq \varepsilon \quad \text { whernever } \quad|t-x| \leq \delta .
$$

Again for $|t-x|>\delta$, then there exist a positive number $M$ such that

Thus for all $t$ and $x \in\left[0, \frac{n}{n+1}\right]$, we get

$$
|\lambda(t-x)| \leq M \leq M \frac{(t-x)^{2}}{\delta^{2}}
$$

Applying $\left\{B_{n}\right\}$ on (3.1), we get

$$
|\lambda(t-x)| \leq \varepsilon+M \frac{(t-x)^{2}}{\delta^{2}}
$$

$$
\begin{gathered}
\left(B_{n}^{*} f\right)(x)=f(x)\left(B_{n}^{*} 1\right)(x)+f^{\prime}(x)\left(B_{n}^{*}(t-x)\right)(x)+\frac{f^{\prime \prime}(x)}{2}\left(B_{n}^{*}(t-x)^{2}\right)(x) \\
+\left(B_{n}^{*}(t-x)^{2} \lambda(t-x)\right)(x) \\
=f(x)+f^{\prime}(x)\left[\frac{n[1-3 x]-2 x}{(n+1)(n+2)}\right] \\
+\frac{f^{\prime \prime}(x)}{2}\left[\frac{n^{3}\left(2 x-x^{2}\right)+n^{2}\left[11 x^{2}-8 x+2\right]+n\left[17 x^{2}-6 x\right]+6 x^{2}}{(n+1)^{2}(n+2)(n+3)}\right] \\
+n\left(1+\frac{1}{n}\right)^{2} e^{-n x} \sum_{k=0}^{\infty} \frac{n^{k} x^{k}}{k !} \int_{0}^{\frac{n}{n+1}} p_{n . k}(t)(t-x)^{2} \lambda(t-x) d t .
\end{gathered}
$$

Multiplying $n$ both side, we get

$$
n\left[\left(B_{n}^{*} f\right)(x)-f(x)\right]=f^{\prime}(x)\left[\frac{n[1-3 x]-2 x}{(n+1)(n+2)}\right] n+\frac{f^{\prime \prime}(x)}{2}\left[\frac{n^{3}\left(2 x-x^{2}\right)+\left[11 x^{2}-8 x+2\right]+n\left[17 x^{2}-6 x\right]+6 x^{2}}{(n+1)^{2}} \quad(n+2)(n+3) \quad n+n R_{n}(t, x)\right.
$$

Here we write, 


$$
\begin{aligned}
n R_{n}(t, x) & =n\left\{\left(1+\frac{1}{n}\right)^{2} e^{-n x} \sum_{k=0}^{\infty} \frac{n^{k} x^{k}}{k !} \int_{0}^{\frac{n}{n+1}} p_{n . k}(t)(t-x)^{2} \lambda(t-x) d t\right\} \\
\left|n R_{n}(t, x)\right| & =\left|n\left\{\left(1+\frac{1}{n}\right)^{2} e^{-n x} \sum_{k=0}^{\infty} \frac{n^{k} x^{k}}{k !} \int_{0}^{\frac{n}{n+1}} p_{n . k}(t)(t-x)^{2} \lambda(t-x) d t\right\}\right| \\
& \leq n\left\{\left(1+\frac{1}{n}\right)^{2} e^{-n x} \sum_{k=0}^{\infty} \frac{n^{k} x^{k}}{k !} \int_{0}^{\frac{n}{n+1}} p_{n . k}(t)\left|(t-x)^{2} \lambda(t-x)\right| d t\right\}
\end{aligned}
$$

Using (3.2) in equation (3.4), we get

$$
\begin{aligned}
\left|n R_{n}(t, x)\right| & \leq n \varepsilon\left(B_{n}^{*}(t-x)^{2}\right)(x)+\frac{n M}{\delta^{2}}\left(B_{n}^{*}(y-t)^{4}\right)(x) \\
& \leq n \varepsilon o\left(\frac{1}{n}\right)+\frac{n M}{\delta^{2}} o\left(\frac{1}{n^{2}}\right) \\
& \leq \varepsilon+\frac{M}{\delta^{2}} o\left(\frac{1}{n}\right)
\end{aligned}
$$

By choosing $\delta=n^{-1 / 4}$, we get that

$$
\begin{aligned}
\left|n R_{n}(t, x)\right| & \leq \varepsilon+\frac{M}{n^{-1 / 2}} o\left(\frac{1}{n}\right) \\
& \leq \varepsilon+M o\left(\frac{1}{\sqrt{n}}\right) .
\end{aligned}
$$

Since $\varepsilon$ is arbitrary and small, we get

$$
\left|n R_{n}(t, x)\right| \rightarrow 0 \text { as } n \rightarrow \infty \text {. }
$$

Using (3.5) in equation (3.3), we get

This completes the proof.

$$
\lim _{n \rightarrow \infty} n\left[\left(B_{n}^{*} f\right)(x)-f(x)\right]=(1-3 x) f^{\prime}(x)+\frac{x(2-x)}{2} f^{\prime \prime}(x)
$$

\section{ACKNOWLEDGMENT}

The authors are thankful to Director of National Institute of Technology, Raipur (C.G.) for encouragement.

\section{REFERENCES}

[1] Deo N. , Noor M.A. and Siddiqui M.A. "On approximation by class of new Bernstein type operators", Appl. Math. Comput., 201 (2008), 604-612.

[2] Kasana H.S. , Prasad G., Agrawal P.N. and Sahai A. "Modified Szậsz operators" Proceeding of International Conference on Mathematical Analysis and its Applications Pergamon Press, 1985, 29-41.

[3] Papanicolau C.G. "Some Bernstein type operators” Amer. Math. Month, 82 (1975) ,674-677.

[4] Singh S.P., "Some problems on approximation of functions by positive linear operators" Ph.D. Thesis, Roorkee University (1982). 